\title{
ECG Signal Quantization Effects in the Analysis of Atrial Fibrillation
}

\author{
C Vayá ${ }^{1}$, JJ Rieta $^{2}$ \\ ${ }^{1}$ Innovation in Bioengineering Group, Universidad de Castilla-La Mancha, Spain \\ ${ }^{2}$ Biomedical Synergy, Universidad Politécnica de Valencia, Spain
}

\begin{abstract}
During last years spectral analysis techniques applied to the ECG have contributed to the knowledge and management of atrial fibrillation $(A F)$. In this matter, it is proved the utility of parameters extracted from the atrial activity (AA) in the time-frequency domain for episode analysis and characterization. These techniques are mostly applied to high resolution 12-lead ECGs. Nevertheless, these high resolution recordings are not always available in the clinical practice and recordings from low resolution acquisition systems, e.g. Holter systems, must be used instead. In this work we analyze the effects of ECG quantization on the AA extraction quality carried out by one of the most recent techniques, independent component analysis (ICA), and the suitability of time-frequency parameters in low resolution recordings.
\end{abstract}

\section{Introduction}

Atrial Fibrillation (FA) is the most common supraventricular tachyarrhythmia encountered in the daily clinical practice. The prevalence of AF is less than $1 \%$ among population under 60 years old, but it increases significantly among those over 70 , approximating to $10 \%$ in those older than 80 [1]. Recently, spectral analysis has been revealed as a useful tool for atrial fibrillation (AF) description [2]. Furthermore, time-domain methods have been successfully applied in the characterization of AF episodes using the surface electrocardiogram (ECG) [3, 4]. The exhaustive analysis of AF episodes requires previously to separate the atrial activity (AA) component from other bioelectric signals that contribute to the ECG formation [5]. Given that the AA and the ventricular activity (VA) overlap spectrally, linear filtering cannot be use to separate them. In consequence, extraction methods based on nonlinear signal processing, as Average Beat Substraction (ABS) [6] or Independent Component Analysis (ICA) [7], must be used instead. Most of times, this nonlinear techniques have been applied to high resolution 12-lead ECGs. Nonetheless, in the particular case of AF, long duration recordings are frequently essential to the correct diagnosis of patients. Be- cause of memory constraints of recorder devices, low resolution acquisition systems, namely Holter systems, have to be usually used. A matter of public interest is to know to which extent low resolution ECGs can be usable to characterize AF episodes in the time-frequency domain. In this essay, we present the effects of progressive decreasing signal resolution in the estimation of time-frequency distributions parameters. The study of these effects are particularized on the main peak frequency $\left(f_{p}\right)$ and the Spectral Concentration $(S C)$. Furthermore, accuracy of the Sample Entropy (SampEn) of the extracted time-domain AA signals for different degrees of quantization has been also analyzed.

\section{Database and methods}

We analyzed a total of 18 ECG signals of sixteen seconds in length, sampling rate equal to 500 samples per second and digitized with 16 bits. All signals were upsampled from $500 \mathrm{~Hz}$ at $1 \mathrm{kHz}$ in order to improve frequency resolution. The respiratory induced axis-shifts of all the analyzed ECG recordings were previously eliminated by high pass filtering and the network interference was canceled by notch filtering. On the contrary, low pass filtering of ECGs was not applied as a previous step to ICA because of the proved higher performance of ICA when post-filtering is used instead of pre-filtering [8]. This fact is explained by the increase of signal gaussianity as a consequence of low pass filtering [9]. Therefore, low pass filtering was applied after ICA to reduce thermal and muscular noise.

The original signals were quantized using from 15 to 4 bits. Then the FastICA [9] algorithm was applied to extract the estimated AA from every signal. the separated signals with lower kurtosis are considered to be the AA sources [7]. The digital post-ICA filtering was Chebyshev type II ninth order with cut-off frequency $70 \mathrm{~Hz}$ and 40 $\mathrm{dB}$ ripple in the stop-band and was applied to all the estimated AA signals . Next, the spectrograms of the filtered AA signals were computed using 1024 points Fast Fourier Transform (FFT), 1024 sample size Hamming window and $75 \%$ overlapping. Besides, time-domain sequences of the AA spectrograms characteristic parameters, i.e. the mean peak frequency $\left(f_{p}\right)$ and the $S C$ ), were constructed. The 
$S C$ is defined as [10]:

$$
S C=\frac{\sum_{f=0.82 f_{p}}^{1.17 f_{p}} P_{A A}(f)}{\sum_{f=0}^{0.5 f_{s}} P_{A A}(f)}
$$

where $P_{A A}$ is the power spectral density of the AA signal, $f$ is the frequency, $f_{s}$ is the sampling rate $(1 k H z)$, and $f_{p}$ is the main frequency peak of the AA. The general process is depicted in figure 1. Furthermore, the SampEn of quantized AA, although not depicted, is also computed to measure the mathematical organization of time-domain signals $[11,12]$.

\section{Performance indexes}

We used two performance indexes to quantify the effects of signal quantization. On the first hand, the crosscorrelation between the AA quantized by 16 bits $\left(A A_{16}\right)$ and the AA quantized by $q$ bits $\left(A A_{q}\right)$ was defined as:

$$
R_{A A}=\frac{\boldsymbol{E}\left\{A A_{16} \cdot A A_{q}\right\}}{\sqrt{\boldsymbol{E}\left\{A A_{16}^{2}\right\} \boldsymbol{E}\left\{A A_{q}{ }^{2}\right\}}}
$$

$R_{A A}$ was obtained as a measure of signal estimation quality since it quantifies the degree of similarity between signals. On the other hand, the Minimum Absolute Error (MAE) index for two vectors $x_{1}$ and $x_{2}$, both of length $N$, is computed as:

$$
M A E=\frac{\sum_{i=1}^{N}\left|x_{1}(i)-x_{2}(i)\right|}{N}
$$

MAE provided a different point of view on the degree of similarity between signals. MAE has the advantage of being measured with the same units than vectors $x_{1}$ and $x_{2}$. MAE was applied to the extracted parameters series, $f_{p}$ and $S C$, and to the SampEn of AA signals as well.

\section{Results}

Figure 2 illustrates the relation of $R_{A A}$ with the number of quantization bits. $R_{A A}$ was computed for both the raw $A A_{q}$ and the low pass filtered $A A_{q}$. It could be observed the better estimation quality when $R_{A A}$ is applied to the low pass filtered $A A_{q}$. In consequence, subsequent computing, including $M A E$ estimation, was applied to the filtered signals. Results revealed that $R_{A A}$ remained higher than 0.7 when the number of quantization bits was higher or equal to nine. For eight or seven bits, $R_{A A}$ approximate to 0.65 , decreasing significantly when the number of bits was lower or equal to six.

On the other hand, the MAE obtained for $f_{p}$ is represented in figure 3. There it is shown that $f_{p} M A E$ did not surpassed $1.3 \mathrm{~Hz}$ when the number of bits was higher or equal to seven but it increased drastically below seven bits. In the same way, as shown in figure $4, S C M A E$ maintained fewer than $6 \%$ when at least seven quantification bits were used, but higher values of $M A E$ were obtained when a more reduced number of bits was considered.

Finally, $M A E$ for $S a m p E n$ of AA signals is depicted if figure 5. SampEn MAE denoted a good approximation of SampEn when seven or more bits were used but it worsens considerably in other case.

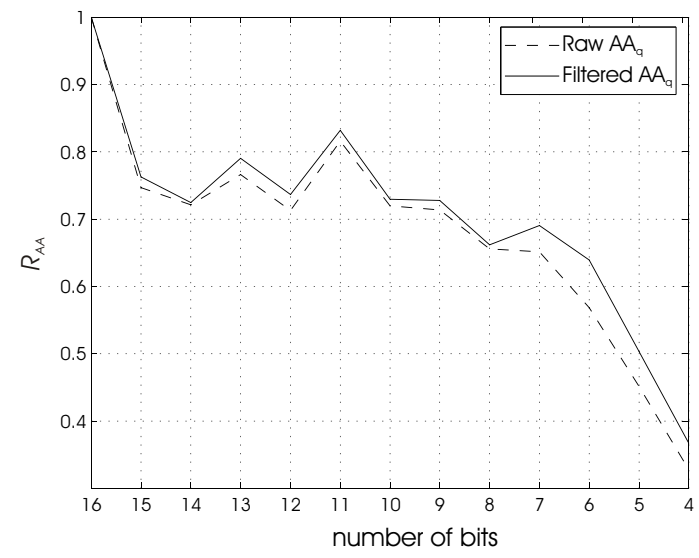

Figure 2. $R_{A A}$ between the 16-bit AA signal $\left(A A_{16}\right)$, and the $\mathrm{AA}$ at the rest of quantization levels $\left(A A_{q}\right)$.

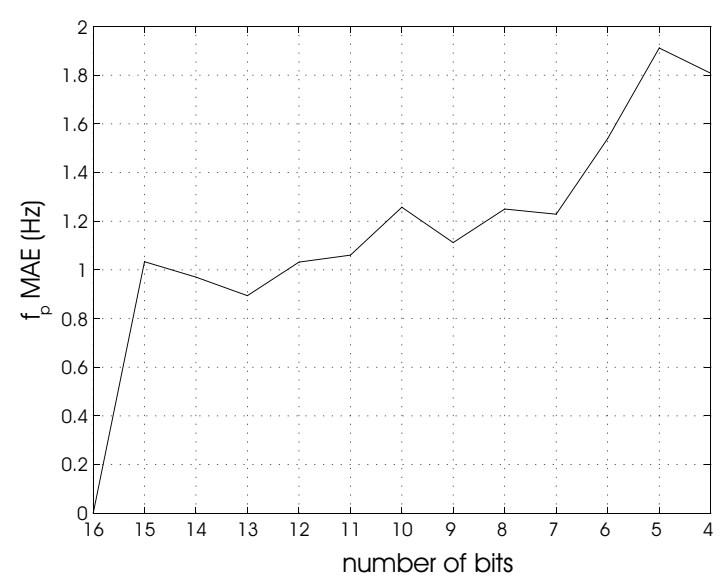

Figure 3. $f_{p} M A E$ for different number of quantization bits. 
(a)

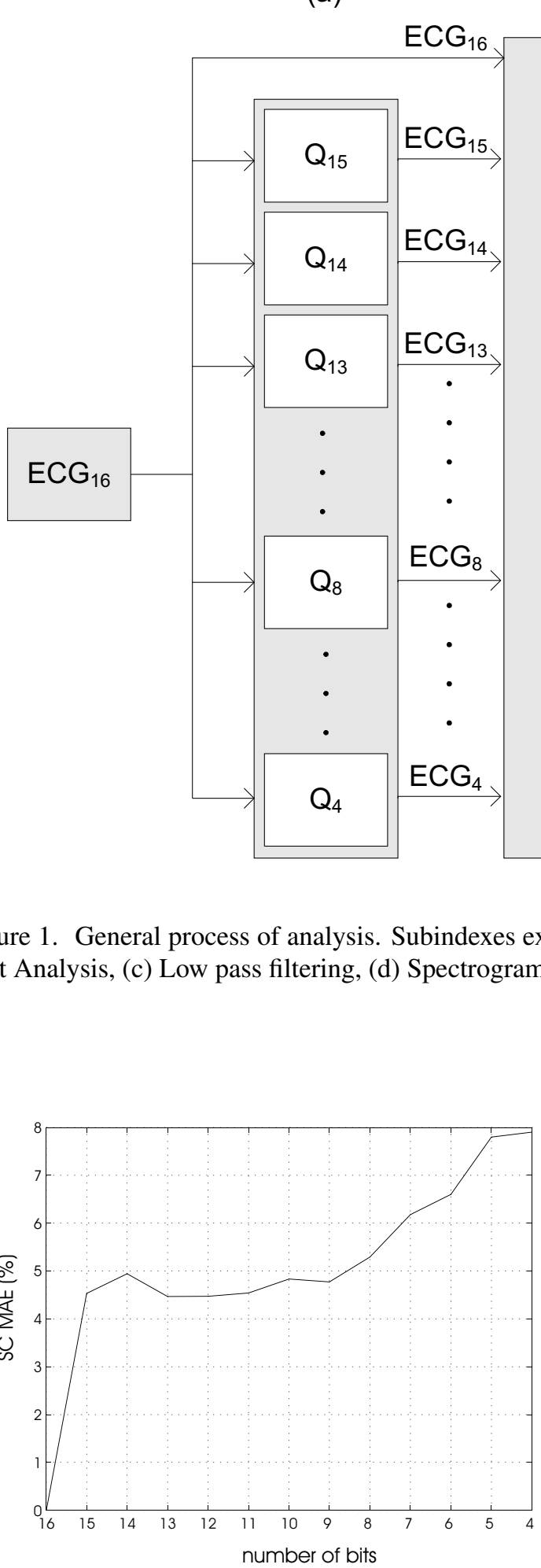

Figure 1. General process of analysis. Subindexes express the number of bits. (a) Quantization, (b) Independent Component Analysis, (c) Low pass filtering, (d) Spectrogram computation and parameter extraction. (b)

(c)

$$
\mathrm{AA}_{16}
$$

$\mathrm{AA}_{15}$

I

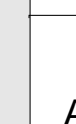

A

C $\quad \cdot \quad$ Butter.

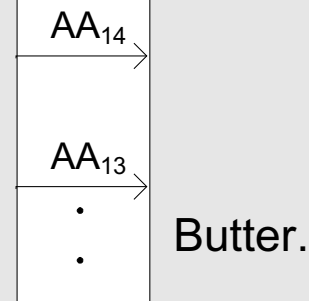

Filter
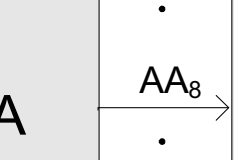

(d)

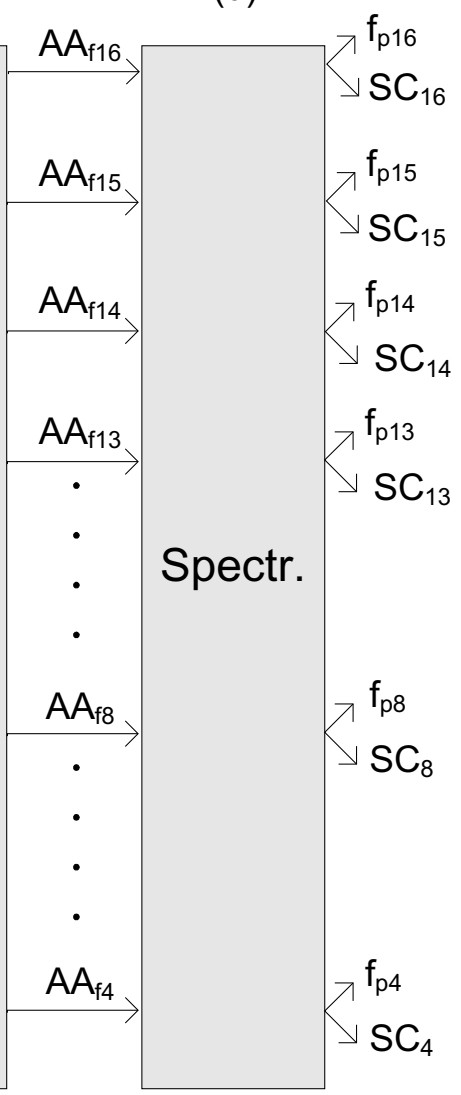




\section{Conclusions}

To sum up, this study revealed that eight bits can be considered as the minimum real resolution threshold than can provide acceptable results. However, this only can be affirmed when the effective dynamic range approximates to the nominal dynamic range. This would require a real-time adjustment of systems that is hardly available in Holter recorders. Therefore, taking into account the headroom margin that has to be considered to avoid recording overflow, the minimum recommended number of bits is twelve.

\section{Acknowledgements}

This work was partly supported by the projects 20070086 from the R+D+i Vice-rectorate of the Valencia University of Technology, GV06/299 from Consellería de Empresa, Universidad y Ciencia de la Generalitat Valenciana and TEC2007-64884 from the Spanish Ministry of Education and Science.

\section{References}

[1] Fuster V, Ryden LE, Asinger RW, et al. CC/AHA/ESC guidelines for the management of patients with atrial fibrillation. Journal of the American College of Cardiology 2001;38(4):1266/I-1266/LXX.

[2] Langley P, Bourke JP, Murray A. Frequency analysis of atrial fibrillation. In Proc.Int.Conf on Computers in Cardiology. 2000; 65-68.

[3] Stridh M, Sörmno L, Meurling C, Olsson B. Characterization of atrial fibrillation using the surface ECG: time-dependent spectral properties. IEEE Transactions on Biomedical Engineering 2001;48(1):19-27.

[4] Husser D, Stridh M, Sörnmo L, Geller C, Klein HU, Olsson SB, Bollman A. Time-frequency analysis of the surface
ECG for monitoring antiarrhythmic drug effects in atrial fibrillation. Amer J Cardiol 2005;95:526-528.

[5] Langley P, Rieta JJ, Stridh M, Millet J, Sörnmo L, Murray A. Comparison of atrial signal extraction algorithms in 12lead ECGs with atrial fibrillation. IEEE Transactions on Biomedical Engineering 2006;53(2):343-346.

[6] Slocum J, Sahakian A, Swiryn S. Diagnosis of atrial fibrillation from surface electrocardiogram based on computerdetected atrial activity. Journal of Electrocardiology 1992; 25(1):1-8.

[7] Rieta JJ, Castells F, Sánchez C, Zarzoso V. Atrial activity extraction for atrial fibrillation analysis using blind source separation. IEEE Transations on Biomedical Engineering 2004;51(7):1176-1186.

[8] Rieta JJ, Sánchez C, Sanchis JM, Castells F, Millet J. Mixing matrix pseudostationarity and ECG preprocessing impact on ICA-based atrial fibrillation analysis. Lecture Notes in Computer Science 2004;3195:1079-1086.

[9] Hyvärinen A, Karhunen J, Oja E. Independent Component Analysis. John Wiley \& Sons, Inc, 2001. ISBN 0-471-405$40-\mathrm{X}$.

[10] Castells F, Rieta JJ, Millet J, Zarzoso V. Spatiotemporal blind source separation approach to atrial activity estimation in atrial tachyarrhythmias. IEEE Transactions on Biomedical Engineering 2005;52(2):258-267.

[11] Pincus SM. Approximate entropy (ApEn) as complexity measure. Chaos 1995;5(1):110-117.

[12] Richman JS, Moorman JR. Physiological time-series analysis using approximate entropy and sample entropy. Am J Physiol Heart Circ Physiol 2000;(278):H2039-H2049.

Address for correspondence:

Carlos Vayá

Innovation in Bioengineering.

Universidad de Castilla-La Mancha.

Camino del Pozuelo, s/n, 16071, Cuenca.

carlos.vaya@uclm.es 